\title{
Internet de las cosas: aplicación en monitoreo de un sistema de generación fotovoltaico
}

\author{
Omar Otoniel Flores-Cortez' \\ Docente Utec \\ omar.flores@utec.edu.sv \\ German Antonio Rosa² \\ Docente Utec \\ german.rosa@mail.utec.edu.sv
}

Recibido: 02/04/2016 - Aceptado: 03/05/2016

\section{Resumen}

En este artículo se presentan los resultados obtenidos de la investigación denominada "Internet de las cosas: interconexión de un sistema de generación fotovoltaico para su monitoreo desde la nube", desarrollada durante 2015 en la Utec, y cuyo objetivo es el diseño e implementación de un sistema electrónico de bajo costo que permitiera el monitoreo vía internet del voltaje producido por un panel solar.

A continuación se describen tanto los procedimientos realizados como los frutos obtenidos a partir de la investigación. Se realizó una revisión teórica del "estado del arte" en cuanto a los conceptos y herramientas tecnológicas utilizados. La metodología usada fue la experimentación-multimodal (Cegarra, 2004); y al final se presentan los resultados obtenidos: el principal, un prototipo electrónico completamente funcional e

\section{Abstract}

This article presents the results of the research called: "Internet of Things: interconnection of a system of photovoltaic generation for monitoring from the cloud", developed during 2015 within the Utec whose main objective was the design and implementation of a lowcost embedded enabling monitoring via Internet voltage produced by a solar panel system.

The following describes both procedures performed and the fruits obtained from the research: a theoretical review of the "state of art" was made in terms of concepts and technological tools used, the methodology used was experimentation-multimodal (Cegarra, 2004), and in the end the results are presented: the main electronic fully functional and implemented on the premises of Utec prototype. These achievements were obtained with great satisfaction and importance,

1 Ingeniero electricista, con maestría en Docencia Universitaria y postgrado en Robótica. Actualmente doctorando en Informática y es docente de la cátedra de Electrónica de la Utec.

2 Ingeniero en Sistemas, técnico en Telecomunicaciones, actualmente docente hora-clase de la Utec. 
implementado en las instalaciones de la Utec. Siendo estos logros de suma satisfacción e importancia, por lo que esta investigación solo es el inicio de una serie de proyectos dentro del área.

\section{Palabras clave}

Internet de las cosas, panel solar, microcontroladores, programación de computadoras, Arduino, internet, Raspberry Pi, sistemas embebidos, sensores, Wi-Fi. so This research is just the beginning of a series of projects in the area.

\section{Keywords}

Internet of things, solar panel, microcontrollers, computer programming, Arduino, internet, Raspberry Pi, embedded systems, sensors, Wi - Fi .

\section{Introducción}

El presente documento es un resumen de los resultados obtenidos a partir de la realización de la investigación sobre el internet de las cosas (IOT, por sus siglas en inglés) y su aplicación para el monitoreo de un sistema de generación fotovoltaica (SGV), cuyo objetivo principal era implementar un sistema de interconexión para el sistema fotovoltaico del Laboratorio de Electrónica de la Utec, que permita realizar un monitoreo en tiempo real, desde la nube ${ }^{3}$, del rendimiento de este sistema, para poder establecer factores de desempeño a lo largo de su tiempo de uso.

Por lo que disponer de un reporte de datos de consumo y generación del sistema fotovoltaico del Laboratorio de Electrónica de la Utec es un paso lógico que sigue a la reciente implementación de dicho sistema. Actualmente se dispone de paneles solares generando energía que se utiliza en la iluminación interna del salón de prácticas del laboratorio, sin embargo, no se cuenta con un monitoreo ni con datos reales de generación que permitan verificar el desempeño y rendimiento del sistema fotovoltaico instalado.

La investigación fue desarrollada usando el método multimodal-experimentación sin hipótesis previa (Cegarra, 2004), además, se clasificó como del tipo investigación aplicada tecnológica (Cegarra, 2004).
Cabe destacar que, durante el proceso desarrollado, en la investigación se analizaron diferentes aspectos técnicos para la implementación; $y$ fue solo la experimentación lo que permitió obtener resultados y diseños satisfactorios. Con esta investigación se obtuvo como resultado un prototipo electrónico funcional que permite que el nivel de voltaje de un panel solar del SGV, instalado en el Laboratorio de Electrónica de la Utec, sea capaz de reportar dentro de un sitio web, y de forma automática y periódicamente, su nivel de voltaje producido durante su operación. Estos datos reportados por el panel se pueden visualizar en tiempo real en forma gráfica en https://sites.google.com/a/mail.utec. edu.sv/labeleutec/panelsolar

\section{Sistema de monitoreo vía internet}

Imagine el siguiente escenario: usted se dirige a su hogar luego de un arduo día de trabajo en la oficina, y solo piensa en prepararse una suculenta cena al llegar a casa, sin embargo, al abrir el refrigerador se da cuenta de que se han agotado casi todas las provisiones (huevos, leche, pan, etc.) iQué decepción! Tendrá que perder tiempo en ir al supermercado de noche a adquirir lo faltante o pedir cena a domicilio. Que útil fuera que el propio refrigerador le "avisara" con antelación, vía internet, mediante su teléfono inteligente, que se han agotado las provisiones y que debe pasar adquiriendo más, evitando así la pérdida de tiempo y dinero.

3 nube 8. f. Inform. Espacio de almacenamiento y procesamiento de datos y archivos ubicado en internet, al que puede acceder el usuario desde cualquier dispositivo. Drae) 
Hoy en día internet se ha convertido en la red casi omnipresente, la disponibilidad en línea de todo tipo de contenido que tiene el usuario es casi ilimitada, además, los tiempos en los cuales esos contenidos están disponibles es casi instantáneo (Mercado, 2011).

Sin embargo, esos contenidos, actualmente, están disponibles porque han sido subidos ${ }^{4} \mathrm{y}$ actualizados por usuarios reales: personas; y estos contenidos son consumidos por usuarios también reales. Sin embargo, el IOT rompe con este paradigma y busca la conectividad y disponibilidad de contenidos entre cosas-usuarios y/o cosas-cosas. Es basándose en esta premisa que los sistemas del IOT buscan que los propios sistemas 0 dispositivos electrónicos (cosas) generen contenido en internet para ser consumido por usuarios, sean estos personas o dispositivos electrónicos.

El IOT es un área aplicada de los sistemas embebidos electrónicos, cuyo objetivo principal es el uso del internet como medio de comunicación de estos sistemas entre sí, esto para intercambio de información sobre su estado de funcionamiento sin necesidad de intervención de un usuario humano (Gustafson, 2014), por lo que el IoT es un área aplicada que busca desarrollar circuitos electrónicos que permitan la conexión de internet a dispositivos o aparatos, con el objetivo de que estos puedan intercambiar datos con otros usuarios de internet (Vega, 2014).

\section{Implementación del sistema de IoT}

Técnicamente existen dos opciones para permitir que un aparato, dispositivo, máquina, etc., pueda estar conectado a internet e intercambiar datos con otros usuarios. La primera es incluir, en el diseño primario y en la fabricación del propio dispositivo, estos circuitos e interfaces que permiten dicha conexión; otra opción es dotar de un circuito externo, posfabricación del dispositivo o máquina, que le brinde la capacidad de comunicación vía internet.

En el caso de esta investigación, se usó la segunda opción, pues el panel solar ya está trabajando y no dispone, de fábrica, de una conexión a internet. Se decidió diseñar un sistema electrónico embebido que se adaptara a la salida de producción de voltaje del panel y que fuera capaz de enviar esta información vía internet a un sitio web para su monitoreo desde cualquier punto con acceso.

Todo el sistema de monitoreo del panel se diseñó en cuatro etapas bien definidas, y son las siguientes:

1. Adquisición de datos de producción del voltaje del panel (hardware: sensor electrónico).

2. Interface de procesamiento y conexión a internet (hardware: microcontrolador).

3. Plataforma informática en internet, para la colección de estos datos (software: servicio de almacenamiento en la nube)

4. Sitio web para la presentación de los datos (software: web hosting de Google Sites).

4 subir 21. tr. Inform. colgar (introducir información en una página web, Drae). 


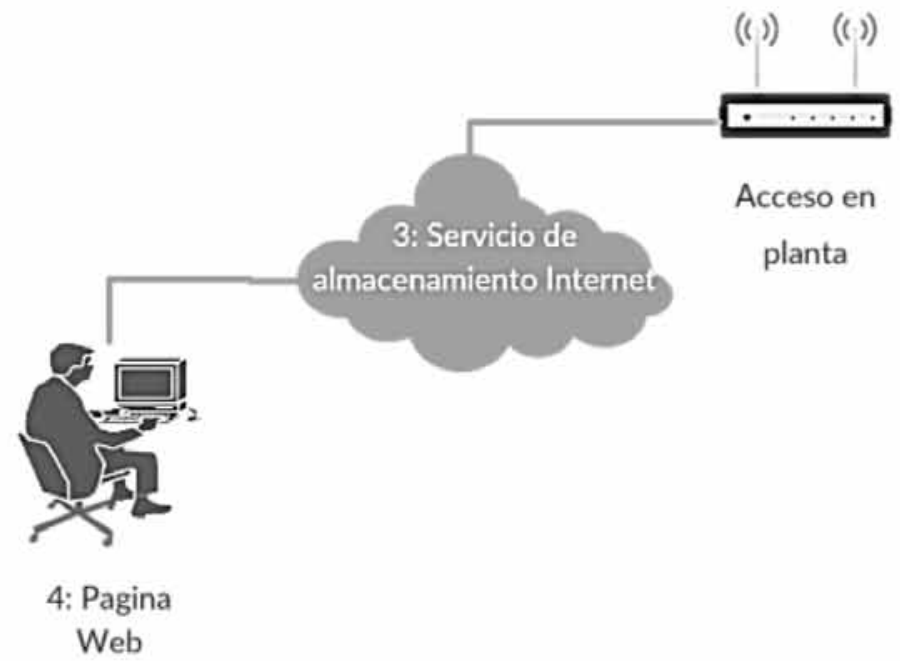

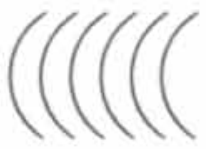

Figura 1

Fuente: Diseño propio.

\section{Herramientas utilizadas}

Para la implementación del sistema diseñado se utilizaron las herramientas de última generación y que, a su vez, son de bajo costo, sin dejar de lado los aspectos como la familiaridad y la experiencia con estas.

Para el bloque de Adquisición de datos se utilizó un sensor de voltaje de tipo activo-análogo apto para la medición de voltaje DC en el rango de 1 a 15 voltios, específicamente se usó el sensor con referencia Pow05161p, cuya salida es un nivel de voltaje apto para ser llevado a la siguiente etapa (Adamo, 2015).

En el bloque de Procesamiento y conectividad se utilizó una tarjeta Arduino modelo YUN, la cual incluye un microcontrolador ATmega32u4 junto con un transceptor o módem para conexiones Wi-Fi (Gómez, 2015). Además se diseñó un firmware en lenguaje Arduino $C$, para que la tarjeta cumpliera la función de procesar los datos del sensor y al mismo tiempo realizará enlaces, vía conexión $W i-F i$, con el servicio de almacenamiento en internet.

Para implementar el tercer bloque del sistema se utilizó la plataforma Temboo (Temboo, 2016), como servicio en la nube, para el almacenamiento en internet de los datos de voltaje producidos por el sistema de procesamiento y adquisición de datos. Esta plataforma permite la creación de una base de datos y una interface entre estos datos almacenados y la página web destinada a la visualización, además Temboo permite el uso de diversas interfaces de programación de aplicaciones (API, por sus siglas en inglés), de las aplicaciones de los servicios de Google, lo cual es muy beneficioso para el sistemas, ya que la Utec cuenta con la plataforma Google para sus servicios estudiantiles y docentes. El servicio de Temboo se programó en conjunto con la plataforma Arduino para capturar, almacenar y transferir la información a través de la API de Google hacia la última etapa del sistema. 
El sitio web para la presentación de los datos de voltaje del panel solar fue diseñado y programado usando la herramienta Google Sites. Como se mencionó anteriormente, se eligió esta opción por ser los servicios que usa la Utec.
El sistema completo fue implementado con éxito en el panel solar del Laboratorio de Electrónica de la Utec. Se programó para generar una medición cada media hora y así poder visualizar cómo cambia su producción de voltaje a lo largo de un día, una semana, una estación, etc.

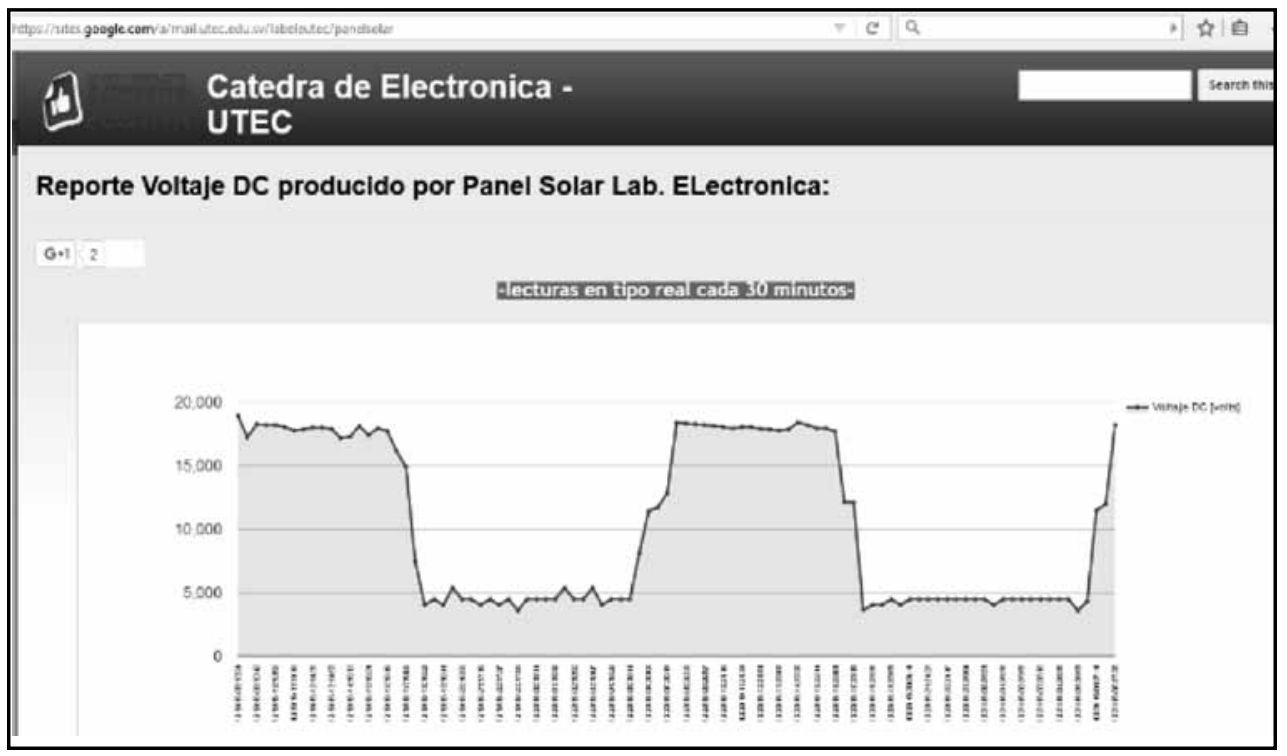

Figura 2

Captura del gráfico de reporte de voltaje producido en un día típico, disponible en el sitio web

Fuente: https://sites.google.com/a/mail.utec.edu.sv/labeleutec/panelsolar

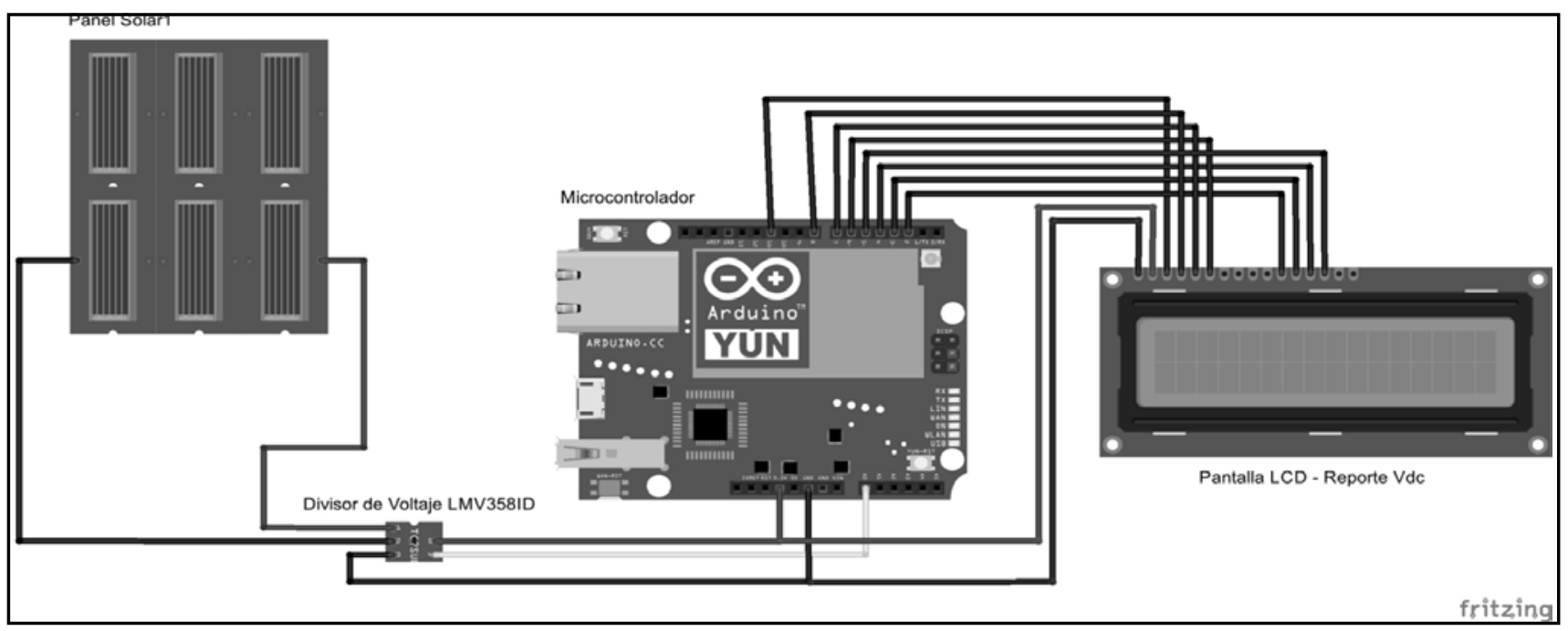

Figura 3

Esquema electrónico de conexión de dispositivo diseñado

Fuente: Diseño propio. 


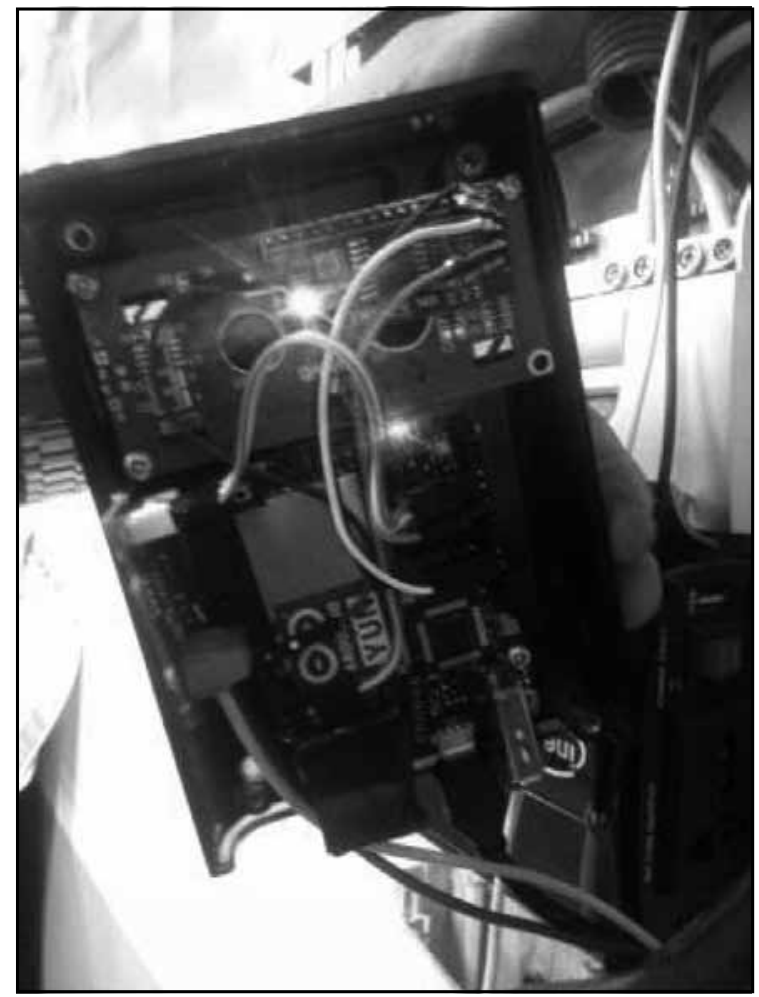

Figura 4

Detalle de la conexión de los componentes del prototipo de monitoreo

Fuente: propia.

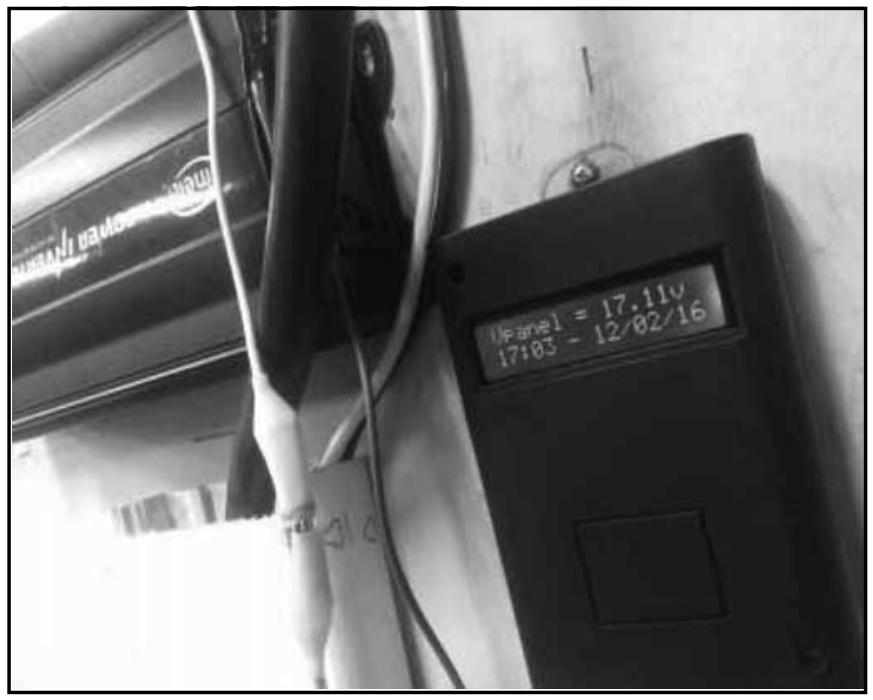

Figura 5

Detalle del prototipo de monitoreo (der.) instalado junto a la acometida del panel solar. (Se puede notar el detalle de la pantalla LCD reportando voltaje) Fuente: Propia. 


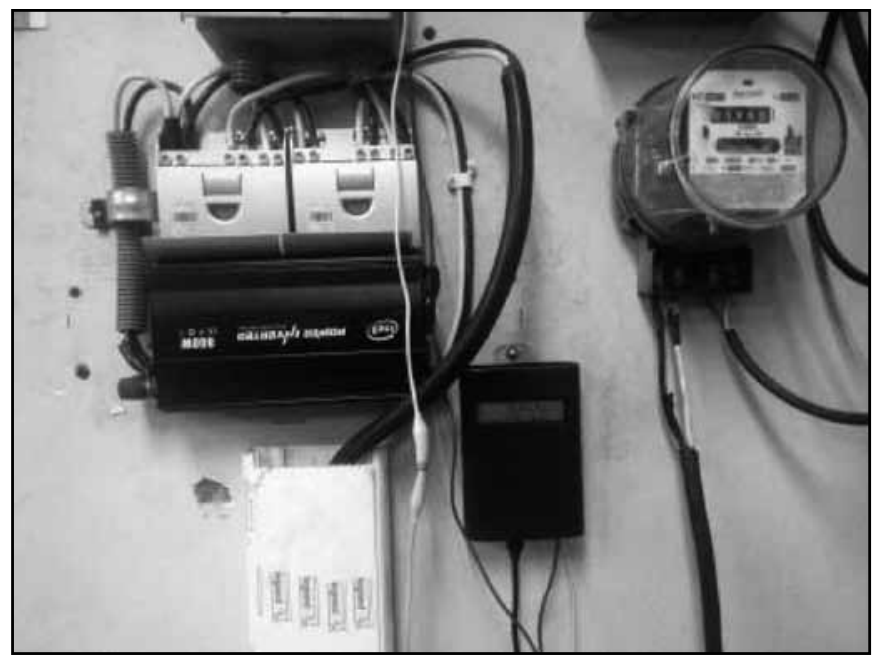

Figura 6

Detalle del Prototipo de Monitoreo (der.) instalado junto a la acometida del panel solar Fuente: propia.

\section{Resultados obtenidos y conclusiones}

El sistema implementado permite que un usuario que acceda a https://sites.google.com/a/mail.utec.edu.sv/ labeleutec/panelsolar pueda observar en tiempo real una gráfica con los datos del voltaje producido por el panel solar experimental del Laboratorio de Electrónica de la Utec. Se muestran en la página anterior algunos gráficos y fotografías del sistema electrónico implementado.

En conclusión, el sistema cumple con el objetivo de permitir al panel solar ser monitoreado desde cualquier punto o dispositivo con acceso a internet, con lo cual se ha demostrado que se pueden implementar soluciones electrónicas de este tipo con el objetivo de monitoreo remoto del desempeño de instalaciones similares a la estudiada en esta investigación. Además se abre la posibilidad de realizar más investigaciones dentro del campo de los sistemas electrónicos embebidos en aplicaciones en el área del IOT.

Los autores agradecen las contribuciones de la Vicerrectoría de Investigación y Proyección Social, al Decanato de la Facultad de Informática y Ciencias Aplicadas y a la Dirección de la escuela de Informática de la Utec.

\section{Referencias}

Adamo, F.; Attivissimo, F.; Cavone, G.; Guarnieri Calo Carducci, C., \& Lanzolla, A. M.L. (2015, May). “New technologies and perspectives for laboratory practices in Measurement science". In Instrumentation and Measurement Technology Conference (I2MTC), 2015 IEEE International (pp. 1-6). IEEE.

Cegarra Sánchez, J. (2004). Metodología de la investigación científica y tecnológica. Madrid, Díaz de Santos.

Gómez Reina, G.A.; Vélez, C., \& Michael, R. (2015). “Telecontrol Domótico de Casa de campo".

Gustafson, S., \& Sheth, A. (2014). Web of Things. Computing Now, 7(3).

Mercado, G.; Diedrichs, A., \& Aguirre, M. (2011). The Wireless Embedded Internet. Annals of CASE.

Temboo, I. (2016). "Temboo". Obtenido de https://temboo.com/ Vega, A.M.; Santamaría, F., \& Rivas, E. (2014). "Internet de los objetos empleando arduino para la gestión eléctrica domiciliaria". Revista EAN (77), 24-40. 